\title{
DLC3 expression in hepatocellular carcinoma
}

\author{
Dominika Wolosz', Grzegorz Szparecki', Ewa Wolinska', Barbara Gornicka' \\ ${ }^{1}$ Department of Pathology, Medical University of Warsaw, Warsaw, Poland
}

Wolosz D, Szparecki G, Wolinska E, Gornicka B. DLC3 expression in hepatocellular carcinoma. J Pre-Clin Clin Res. $2015 ; 9(2)$ : $105-108$. doi: $10.5604 / 18982395.1186488$

\section{Abstract}

Introduction and objective. Deleted in Liver Cancer (DLC) proteins belong to the family of RhoGAPs and thus are believed to operate as negative regulators of the Rho family of small GTPases. Rho proteins are considered to be significant links between numerous cellular pathways. So far, DLC1 - the first identified member from Deleted in Liver Cancer family - has been established as a tumour suppressor in hepatocellular carcinoma. As shown by many studies, DLC1 expression is reduced by gene loss or epigenetic silencing in this type of cancer. The expression and function of its close family relative DLC3 is less known. The presented study determined the expression and cellular localization of DLC3 protein in hepatocellular carcinoma tissue.

Materials and methods. The protein level in two types of hepatocellular carcinoma: typical and fibrolamellar, were assessed by the immunohistochemical approach.

Results. DLC3 immunoreactivity was found to be present in the cytoplasm of normal hepatocytes. In hepatocellular carcinoma sections, DLC3 was detected primarily in the cytoplasm of cancer cells, although in a small percentage of cancer cells cell nuclei were also positively stained. Morphometric analysis followed by statistical evaluation showed that the DLC3 immunoreactivity in the tumour sections was comparable to the one observed in non-cancerous liver specimens.

Conclusions. The results obtained indicate that DLC3 protein, contrary to DLC1, is commonly expressed in hepatocellular carcinoma. It appears that members of the DLC family, although structurally highly related, may function differently during HCC development.

\section{Key words}

DLC3, hepatocellular carcinoma, fibrolamellar hepatocellular carcinoma

\section{INTRODUCTION}

Rho proteins serve as key regulators of numerous signaling pathways that regulate such cellular processes as proliferation, apoptosis, motility, gene expression and reorganization of the actin cytoskeleton [1]. Due to their ability to control a wide range of cellular functions, deregulation of their activities may contribute to various aspects of tumourigenesis.

Rho proteins function as molecular switches, oscillating between an active GTP-bound and an inactive GDP-bound state. The exchange between GDP and GTP is controlled, among others, by the family of GTPase activating proteins (GAPs) which catalyze GTP hydrolysis to restore the inactive form [1]. As numerous studies have shown, Rho signaling pathways are often altered in human cancers [2]. One of the major causes of these alterations is the malfunction of GTPase activating proteins.

DLC1, DLC2 and DLC3 (Deleted in Liver Cancer) proteins belong to the RhoGAP super family. The best characterized member of this family, $D L C 1$, was found to be frequently deleted in different types of cancer, and its tumour suppressive function has been clearly evidenced [3].

The least characterized member of the DLC family is DLC3, a gene localized in the Xq13 chromosome. The first report depicting its structure and function revealed a high similarity to the other two proteins [4]. Similarly to DLC2, DLC3 was found to be present in focal adhesion sites and to affect cell morphology [5]. It was demonstrated that the over-expression of DLC3 inhibited cell proliferation, colony forming ability and growth in soft agar in human breast and prostate cancer

Address for correspondence: Ewa Wolinska, Department of Pathology, Medical University, Warsaw, Zwirki i Wigury 61, 02-091 Warsaw, Poland

E-mail: ewa.wilczek@wum.edu.pl

Received: 30 October 2015; accepted: 2 December 2015 cell lines [4]. Like its relatives, DLC3 was shown to be downregulated in a significant fraction of primary kidney, lung, ovary and breast tumours [4].

Liver cancer is the fifth most common form of cancer and the third leading cause of deaths worldwide. [6] Hepatocellular carcinoma represents the most common primary liver cancer among adults. It develops predominantly in patients with underlying cirrhotic liver disease of various etiologies [7]. Although a great of progress in the understanding of cancer biology has been made in the last few decades, the pathophysiology of HCC is still poorly characterized. As many studies highlighted, HCC is a complex disease, thus attempts to use molecular therapy are beneficial only in a small cohort of patients [8]. To-date, the best treatment options remains tumour resection; however, liver transplantation, chemoembolization and thermoablation may also improve overall survival [9].

Within the heterogenous group of hepatocellular cancer types there is one subgroup - fibrolamellar (FL) HCC, a rare form of this primary liver cancer which typically affects young adults. FL HCC has an unknown etiology, and in most patients with this subtype of carcinoma no underlying liver cirrhosis or other concurrent liver disease is present [10].

\section{OBJECTIVE}

The aim of the presented study was to determine the presence and tissue/cellular localization of the DLC3 protein in HCC tumour samples. Among the experimental group there was also a group of FL subtype of HCC. To the best of the knowledge of the authors of the current study, this is the first study to assess the expression of DLC3 protein in this type of tumour. 


\section{MATERIALS AND METHOD}

Tissue specimens. Tissue samples were obtained from 81 patients with diagnosed HCC. Among this group, 9 represented the fibrolamellar subtype. Additionally, 25 normal liver tissues were included as a control group.

Ethics statement. The study was approved by the Ethics Committee of the Medical University in Warsaw (No. $\mathrm{KBO} / 42 / 11)$.

Immunohistochemistry/Immunofluorescence. In brief, the immunohistochemical staining was as follows: formalinfixed, paraffin-embedded sections were deparaffinized, blocked with 3\% hydrogen peroxide and with 5\% normal donkey serum (Jackson Immunoresearch, USA). After such blocking, the sections were treated with high temperature boiling in citrate buffer to unmask antigen sites. Subsequently, rabbit anti-DLC3 (ProteinTech, USA) antibodywas applied. In a negative control reaction, normal rabbit IgG in the same concentration as the primary antibody was applied. After incubation, detection was performed with donkey anti-rabbit peroxidase-conjugated (Jackson Immunoresearch) antibody and 3, 3'-diaminobenzidine (Dako, Denmark) used as a chromogen.

Morphometric analysis. DLC3 immunoreactivity was quantified by morphometric analysis using a Nikon Eclipse 80i microscope and Image Pro Plus software. From each patient, 10 random fields were photographed at 20x magnification. On every the area covered by the immunoreactivity, as well as the mean intensity of staining, was quantified. Within a given field, the product of immunoreaction intensity times the area was considered as an approximation of the total immunoreactivity, and displayed in arbitrary units.

Statistical analysis. Differences between groups were analyzed by the non-parametric Mann-Whitney $U$ test. Statistical correlations were evaluated by the Spearman's rank correlation coefficient test.

\section{RESULTS}

DLC3 immunoreactivity in the normal liver was detected in the cytoplasm of virtually all hepatocytes, in the form of diffused, intense staining (Fig. 1A). A mild DLC3 immunoreactivity in hepatocytes nuclei was also observed.

In the $41 \mathrm{HCC}$ specimens examined, similarly to the liver tissues, DLC3 immunoreaction was found to be mainly localized in the cytoplasm of cancer cells (Fig. 1B-D); however, nuclear presence was also seen (Fig. 1E). In the majority of tumour samples, a strong to moderate intensity of the immunoreactivity was observed. Morphometric analysis of DLC3 immunoreactivity followed by the statistical analysis showed that the DLC3 protein level in HCC sections was comparable to that observed in non-cancerous liver tissues. Similarly, no differences were observed in the DLC3 immunostaining pattern between the fibrolamellar variant of HCC and its typical subtype (Fig. 1D). The negative control staining, performed with the use of normal rabbit IgG applied instead of the primary antibody, showed no immunoreactivity
(Fig. 1F). No statistically significant correlation was detected between DLC3 protein expression and any of the clinical parameters of HCC patients.

\section{DISCUSSION}

The current study documents the presence of DLC3, a member of the DLC family protein in hepatocellular carcinoma, and presents novel results which indicate that this protein, unlike its close relatives, is present in hepatocellular carcinoma tissues. Additionally, no differences were detected between two types of hepatocellular carcinoma - the classical and fibrolamellar variants. The results obtained indicate that the least known member of the Deleted in Liver Cancer family of proteins, namely DLC3, is expressed at the similar level both in normal and transformed cells.

To-date, the sub-cellular localization of DLC3 protein has been poorly characterized. By the immunofluorescent approach, Kawai et al. showed that DLC3 was observed in focal adhesions [5]. In the presented study, DLC3 protein immunoreactivity was detected in the cytoplasm of cancer cells; however, in a small percentage of tumours, cell nuclei were also positively stained. Recently, it was shown that DLC1 localized in the nuclei has reduced the tumour suppressive function [11]. It can be assumed that the nuclear translocation of DLC3 may represent a mechanism of its activity reduction in these cancer cells which possess an intact DLC3 expression. On the other hand, it is intriguing to speculate whether the nuclear localization of DLC3 has anti-oncogenic potential. In in vitro assay, the DLC3 protein demonstrates substrate specificity toward RhoA and interacts with tensin proteins in focal adhesions [5]. Recent publications have shown that Net1, a RhoA specific GEF, localizes to the nucleus and activates the nuclear pool of RhoA after DNA damage signals [12]. This is the first report regarding RhoA nuclear translocation and its activation after the application of DNA damaging agents. Hence, a plausible explanation for the presence of DLC3 in the nucleus includes regulation of the mechanism of the Rho-mediated signaling processes Furthermore, other focal adhesion proteins have been found in the nucleus, where they have been involved in the controlling of gene expression [13]. It will be of particular interest to define whether the nuclear localization of DLC3 protein is accompanied by RhoA nuclear export, and to assess the biological stimulus that causes translocation of DLC3 from the cytosol to the nucleus in HCC.

In the presented study, no differences were observed in the immunoreactivity in normal vs. tumour cells. However, as shown by Durkin et al., the DLC3 gene has an alternative transcript, DLC $3 \alpha$ and two additional isoforms, DLC3 $\beta$ - which lacks the SAM domain, and a highly truncated DLC $\gamma$ [4]. DLC3a is thought to be the most prominent isoform, the over-expression of which in cancer cell lines can inhibit cell proliferation. To the best of the knowledge of the authors of this study, there are no specific antibodies available which could detect these DLC3 isoforms; therefore, possible differences in immunoreactivity and the expression pattern of individual isoforms cannot be exactly determined.

It is possible that during $\mathrm{HCC}$ regulation, point mutations of the DLC3 gene may occur, affecting the protein function, while the protein expression may remain intact. In fact, in a large scale analysis of mutations in human breast and colon 

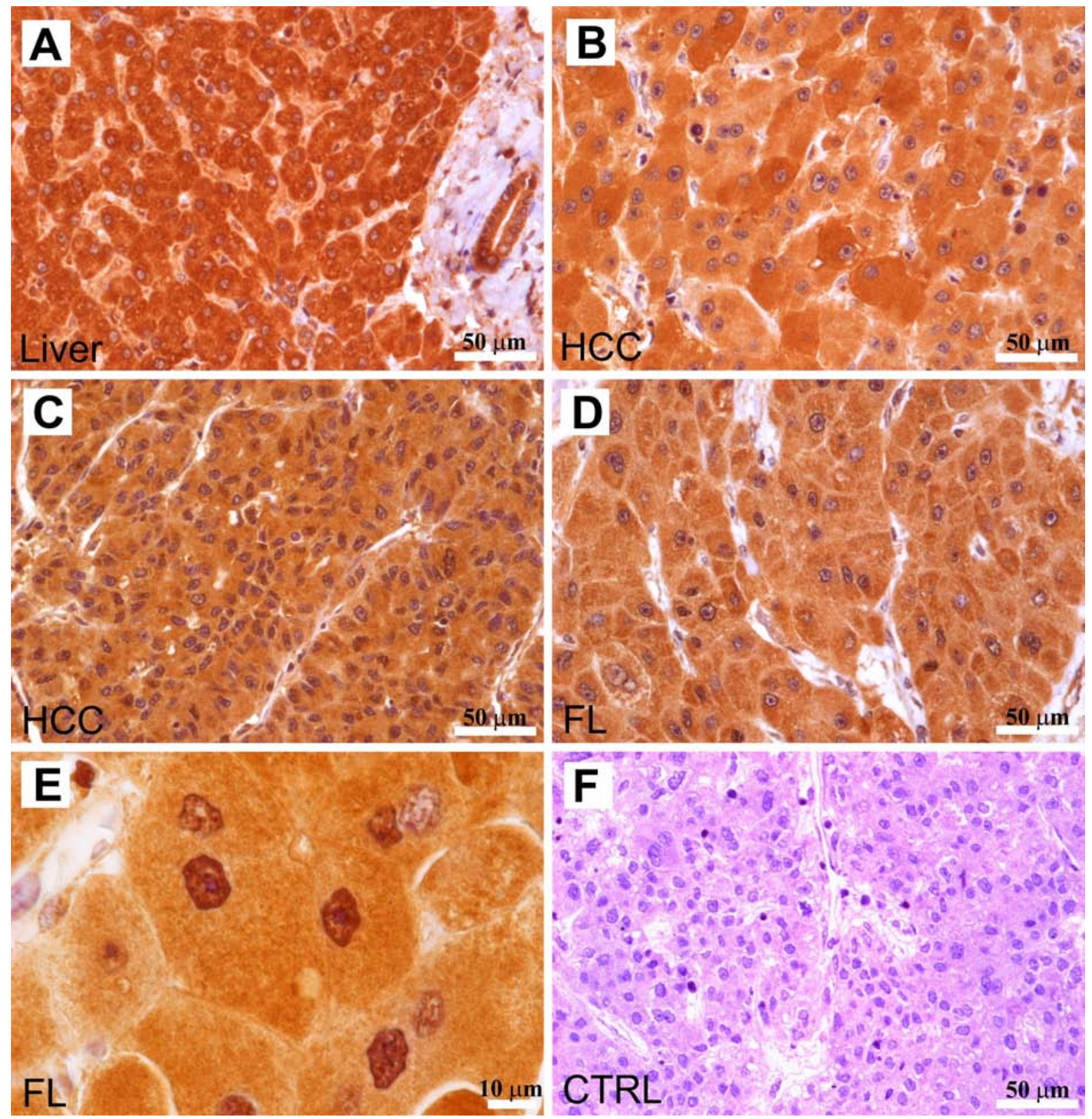

Figure 1. DLC3 immunoreactivity in non-cancerous liver and hepatocellular carcinoma.

A - strong staining in the normal liver; $\mathrm{B}$ - HCC with heterogeneous staining;

$\mathrm{C}$ - homogeneous immunoreaction in HCC exhibiting trabecular pattern; D - FL with moderate staining; $\mathrm{E}$ - cancer cells showing both cytoplasmic and nuclear staining;

$\mathrm{F}$ - negative control

cancers, two missense mutations in DLC3 were found in breast tumours [14]. Whether these type of alterations can be found and affect DLC3 activity in HCC remains to be established.

\section{CONCLUSIONS}

This study demonstrates that DLC3 expression is not diminished in HCC, and the results obtained question the DLC3 oncosuppressive action in this type of cancer. More detailed analysis on the mechanisms which regulate DLC3 activity in the normal development and in pathologic conditions is required to elucidate its contribution to HCC initiation and progression. The study also defines for the first time the expression level of DLC3 in normal liver and in HCC. Since the potential impact of DLC3 on HCC development and progression has not been investigated, it would be interesting to generate mice that lack the DLC3 gene in order to clarify DLC3 protein function in malignant transformation.

\section{Acknowledgements}

The presented work was supported by Grant No. 3509/B/ P01/2010/38 from the Polish State Committee for Scientific Research and by funding from the Medical University of Warsaw, Grant No. 1M11/PM11. 


\section{REFERENCES}

1. Heasman SJ, Ridley AJ. Mammalian Rho GTPases: new insights into their functions from in vivo studies. Nat Rev Mol Cell Biol. 2008; 9: $690-701$.

2. Ellenbroek SI, Collard JG. Rho GTPases: functions and association with cancer. Clin Exp Metastasis. 2007; 24: 657-672.

3.Zimonjic DB, Popescu NC. Role of DLC1 tumour suppressor gene and MYC oncogene in pathogenesis of human hepatocellular carcinoma: potential prospects for combined targeted therapeutics (review). Int J Oncol. 2012; 41: 393-406.

4.Durkin ME, Ullmannova V, Guan M, Popescu NC. Deleted in liver cancer 3 (DLC-3), a novel Rho GTPase-activating protein, is downregulated in cancer and inhibits tumour cell growth. Oncogene 2007; 26: 4580-4589.

5. Kawai K, Kiyota M, Seike J, Deki Y, Yagisawa H. START-GAP3/DLC3 is a GAP for RhoA and Cdc42 and is localized in focal adhesions regulating cell morphology. Biochem Biophys Res Commun. 2007; 364: 783-789.

6. Leonardi GC, Candido S, Cervello M, Nicolosi D, Raiti F, Travali S, et al. The tumour microenvironment in hepatocellular carcinoma (review) Int J Oncol. 2012; 40: 1733-1747.
7. Sanyal AJ, Yoon SK, Lencioni R. The etiology of hepatocellular carcinoma and consequences for treatment. Oncologist 2010; 15 Suppl 4: 14-22.

8. Llovet JM, Ricci S, Mazzaferro V, Hilgard P, Gane E, Blanc JF, et al. Sorafenib in advanced hepatocellular carcinoma. N Engl J Med. 2008; 359: 378-390.

9. Llovet JM, Bruix J. Novel advancements in the management of hepatocellular carcinoma in 2008. J Hepatol. 2008; 48 Suppl 1: S20-37.

10. Kakar S, Burgart LJ, Batts KP, Garcia J, Jain D, Ferrell LD. Clinicopathologic features and survival in fibrolamellar carcinoma: comparison with conventional hepatocellular carcinoma with and without cirrhosis. Mod Pathol. 2005; 18: 1417-1423.

11. Chan LK, Ko FC, Sze KM, Ng IO, Yam JW. Nuclear-targeted deleted in liver cancer 1 (DLC1) is less efficient in exerting its tumour suppressive activity both in vitro and in vivo. PLoS One 2011; 6: e25547.

12. Dubash AD, Guilluy C, Srougi MC, Boulter E, Burridge K, Garcia-Mata $\mathrm{R}$. The small GTPase RhoA localizes to the nucleus and is activated by Net1 and DNA damage signals. PLoS One 2011; 6: e17380.

13. Hervy M, Hoffman L, Beckerle MC. From the membrane to the nucleus and back again: bifunctional focal adhesion proteins. Curr Opin Cell Biol. 2006; 18: 524-532.

14. Sjoblom T, Jones S, Wood LD, Parsons DW, Lin J, Barber TD, et al. The consensus coding sequences of human breast and colorectal cancers. Science 2006; 314: 268-274. 